Spencer, C. P. (1957). J. gen. Microbiol. 16, 282-285

\title{
Utilization of Trace Elements by Marine Unicellular Algae
}

\author{
By C. P. SPENCER \\ Marine Biology Station, University College of North Wales
}

The relationship between the supply of trace elements and the growth of phytoplankton can be considered from many different aspects. The supply of an essential trace element may limit the growth of the unicellular algae by limiting the growth rate, the total crop or both. The physical or chemical form in which the element is present may also be of importance in governing its availability to the organism. The mere demonstration of an absolute requirement for a particular trace element is a technically difficult matter by direct means. In recent years, however, the value of chelating agents in trace element studies has been pointed out (Hutner, Provasoli, Schatz \& Haskins, 1950) and these workers and others have used chelating agents both for the qualitative demonstration of trace element requirements and for some quantitative measurements (Hutner, 1948; Walker, 1953; 1954). Considerable amounts of physico-chemical data are now available concerning the equilibria between certain chelating agents and free metal cations; such data enables these techniques to be much more fully exploited than hitherto. It is the purpose of this communication to describe some recent results which have been obtained during an investigation of the effects of ethylenediamine tetra-acetic acid (EDTA) on the growth of a marine unicellular alga and to discuss the possible implications of the results on various aspects of trace element utilization.

When EDTA is added to sea water a large number of equilibria are set up in which the cations present compete for the available EDTA. In addition, four proton association equilibria (corresponding to the stepwise association of a weak quadribasic acid) must be considered. A general formula has been developed for dealing with multi-equilibria systems of this type. This avoids manipulating the large numbers of simultaneous equations which are needed to deal quantitatively with such systems. In its simplest form the general formula is applicable to a system of any number of competing equilibria involving 1:1 chelate formation between metal and chelating agent. The formula is easily extended to include the formation of chelates of other structure.

By applying the general formula to solutions of various strengths of EDTA in sea water it is possible to calculate the concentrations of all the biologically important cations which can exist in equilibrium with the chelating agent. Because of the great stability of most chelates of EDTA and the very small amounts in sea water of many of the trace elements it is possible to produce culture media in which the concentration of the free ions of many metals is extremely low. This has been realized by the previously mentioned workers and it has become the usual practice to add relatively large amounts of trace 
elements to such media to compensate for this effect. The composition of these media, however, is usually such that the addition of a certain quantity of a trace element causes a disproportionately small increase in the concentration of the free ions of that metal. Calculations show that the concentration of free ions of many metals in typical media of this type is of the order of $10^{-9} \mathrm{M}$ and below.

That many of the unicellular algae will grow at optimum rates in medium of this composition raises interesting problems about the mechanism of utilization of trace elements from such equilibrium mixtures. Two possibilities suggest themselves. First, it is possible that the chelated metal is made available as a result of the organic moiety of the complex being metabolized and the metal being liberated in ionic form. Alternatively, it is possible that the organisms are capable of mobilizing the very low concentrations of free ions the concentration of which will tend to be maintained by some of the complex dissociating to restore the equilibrium. The systems set up in EDTA medium are capable of experimental investigation. Either one can examine the effect of chelation on the toxicity of a heavy metal ion or, alternatively, the effects of chelation on the growth-promoting activity of an essential trace element can be considered. In either case the biological activity might be expected to be related to the concentration of the particular form or forms in which it is available to the organism.

The results reported have been obtained using bacteria-free cultures of a marine unicellular alga Phaeodactylum tricornutum. Culture methods and measurements of growth, with certain minor modifications to be described elsewhere, have been by the techniques previously developed for use with this organism (Spencer, 1954). The cupric ion is increasingly toxic to the test organism at concentrations in excess of about $2 \times 10^{-6} \mathrm{M}$. 'The Cu-EDTA complex does not become toxic until concentrations in excess of $5 \times 10^{-3} \mathrm{M}$ are reached. Calculation shows that the toxicity of the cupric chelate is probably accounted for by the equilibrium concentrations of cupric ions which, under these conditions, are of the order required to cause a comparable growthinhibition in the absence of EDTA.

Similar results are obtained when the effects of EDTA on the availability of manganese are investigated. By prolonged cultivation at high EDTA concentrations it is possible to obtain an inhibition of growth which is reversed by the addition of manganese. Under these conditions the growth-promoting activity of additions of manganese can be shown to be strictly related to the concentration of ionic manganese and to be independent of the total manganese concentration or the concentration of Mn-EDTA complex. These results are in accord with the generally accepted fact that EDTA is not readily metabolized.

The results further indicate that the organisms must be capable of mobilizing manganese ions at an optimum rate at a concentration of about $2 \times 10^{-9} \mathrm{M}-\mathrm{Mn}^{++}$. The results with $\mathrm{Cu}$-EDTA complex suggest that chelated copper is similarly biologically inactive. The concentration of cupric ions existing in the medium used for the manganese studies can be shown to be of the order of $10^{-14} \mathrm{M}$. Copper is generally supposed to be an essential trace element for growth and 
yet no evidence of any copper-deficiency symptoms has been obtained even after several months of cultivation under these conditions. Similar considerations apply to other trace elements, particularly zinc and cobalt. The concentrations of these elements in ionic form in the medium used is of the order of $10^{-13} \mathrm{M}$ and $10^{-14} \mathrm{M}$, respectively. Evidently a 'trace element free' sea water will have to be prepared before the utilization of these elements can be investigated in the same way as manganese.

By analogy with the results obtained with manganese it seems reasonable to expect that the utilization of copper, zinc and cobalt from such equilibrium mixtures will follow a similar pattern. A strong suggestion that the critical concentration for the mobilization of these ions at optimum rate may well be below the concentrations obtained in the present studies can be seen from the results reported by Walker (1953). This worker was apparently able to obtain optimum growth of Chlorella in an EDTA medium which had been prepared by techniques which seem to preclude chance contamination with appreciable quantities of trace elements. It can be shown that the ionic concentrations in Walker's complete medium were of the order of $10^{-9} \mathrm{M}-\mathrm{Mn}^{++} ; 10^{-11} \mathrm{M}-\mathrm{Zn}^{++}$; $10^{-14} \mathrm{M}-\mathrm{Cu}^{++}$. Inhibition of growth only occurred when the concentration of one or other of these ions was lowered by decreasing the total concentration of the particular metal.

It therefore begins to appear that at least some of the unicellular algae may be able to mobilize their essential trace elements from very low ionic concentrations. This possibility may have important implications when we consider various aspects of trace element utilization and their possible role as a regulating factor of the growth of phytoplankton in nature.

The problem of the supply of metals when present as sparingly soluble hydroxides has troubled algologists for many years. The amount of iron which can exist in true solution in sea water at $\mathrm{pH} 8$ is exceedingly small and apparently cannot exceed a concentration of $c .10^{-14} \mathrm{M}$ (Cooper, 1937). The usual practice is to add iron to culture media in complex form in order to prevent the precipitation of ferric hydroxide. Many marine algae can at least partially satisfy their iron requirements when an iron compond is supplied to them largely in some particulate form; it has been suggested that direct utilization of particulate ferric hydroxide can in some cases occur (Harvey, 1937). Nevertheless, even in these cases the marked superiority as an iron source of complex soluble forms can be demonstrated. It is obvious that any complex of iron which will prevent the precipitation of ferric hydroxide must maintain in solution a lower concentration of ferric ions than exists in equilibrium with solid ferric hydroxide. It is beginning to appear increasingly unlikely that the ferric-EDTA chelate will be metabolized. A suggestion has recently been made that even the ferric citrate complex may not be metabolized (Goldberg, 1952). In medium containing iron in complex form, it therefore seems most likely that, as in the case of manganese, the organisms mobilize the ferric ions. The superiority of complexes as an iron source might therefore be due to the fact that the adjustment of the equilibrium subsequent to the removal of ions from such a system is instantaneous. This contrasts to the finite time required for 
the corresponding adjustment to occur by solution of a solid phase. Similar considerations may of course apply to the observed beneficial effects on the availability of trace elements for higher plants when chelating agents are added to soil.

If we consider the trace element supply to the phytoplankton in nature, a certain analogy with the position in EDTA medium is apparent. In both cases the organisms are presented with a large total amount of a particular trace element but at a low immediately available concentration. Except in the case of self-contained bodies of water or perhaps in conjunction with high concentrations of nitrogen and phosphorus when very heavy growths may occur, it seems unlikely that the growth of the phytoplankton will seriously decrease the total amount of a particular trace element. With the more usual relatively sparse crops it has always seemed that the most likely way in which a trace element deficiency might occur in nature was by the concentration of a particular element becoming decreased below the concentration at which it can be mobilized at an optimum rate. The very low concentrations at which this would become important, at least with some organisms, therefore seems to suggest that trace elements are unlikely to have such a regulating effect. The results emphasize even more the generally recognized importance of the form in which the main store of trace elements is present and in particular emphasize the importance of the nature of the equilibrium between the store and the supply of ions.

\section{REFERENCES}

Cooper, L. H. N. (1937). Some conditions governing the solubility of iron. Proc. Roy. Soc. B, 124, 299.

Goldberg, E. D. (1952). Iron assimilation by marine diatoms. Biol. Bull., Woods Hole, 102, 243.

Harvey, H. W. (1937). The supply of ions to diatoms. J. mar. biol. Ass. U.K. 22, 205.

HUtNer, S. H. (1948). Essentiality of constituents of sea water for growth of a marine diatom. Trans. N.Y. Acad. Sci. 10, 136.

Hutner, S. H., Provasoli, L., Schatz, A. \& Haskins, C. P. (1950). Some approaches to the study of the role of metals in the metabolism of micro-organisms. Proc. Amer. phil. Soc. 94, 152.

Spencer, C. P. (1954). Studies on the culture of a marine diatom. J. mar. biol. Ass. U.K. 33, 265.

WALKer, J. B. (1953). Inorganic micronutrient requirements of Chlorella. I. Requirements for calcium (or strontium), copper, and molybdenum. Arch. Biochem. Biophys. 46, 1.

WALKER, J. B. (1954). Inorganic micronutrient requirements of Chlorella. II. Quantitative requirements for iron, manganese and zinc. Arch. Biochem. Biophys. $54,1$. 